\title{
A Simple Network Showing Burst Synchronization without Frequency Locking
}

\author{
Christof Koch \\ Computation and Neural Systems Program, California Institute of Technology, \\ Pasadena, CA 91125 USA

\section{Heinz Schuster} \\ Institut für theoretische Physik, Universität Kiel, \\ Olshausenstrasse 40, 2300 Kiel 1, Germany
}

The dynamic behavior of a network model consisting of all-to-all excitatory coupled binary neurons with global inhibition is studied analytically and numerically. We prove that for random input signals, the output of the network consists of synchronized bursts with apparently random intermissions of noisy activity. We introduce the fraction of simultaneously firing neurons as a measure for synchrony and prove that its temporal correlation function displays, besides a delta peak at zero indicating random processes, strongly dampened oscillations. Our results suggest that synchronous bursts can be generated by a simple neuronal architecture that amplifies incoming coincident signals. This synchronization process is accompanied by dampened oscillations that, by themselves, however, do not play any constructive role in this and can therefore be considered to be an epiphenomenon.

\section{Introduction}

Recently synchronization phenomena in neural networks have attracted considerable attention. This was mainly due to two experimental observations. First, Gray et al. (1989), Engel et al. (1990), as well as Eckhorn et al. (1988) (see also Freeman 1978; Wilson and Bower 1991) provided electrophysiological evidence that neurons in the visual cortex of cats discharge in a semisynchronous, oscillatory manner in the $40 \mathrm{~Hz}$ range and that the firing activity of neurons up to $10 \mathrm{~mm}$ away is phase-locked with a mean phase shift of less than $3 \mathrm{msec}$. It has been proposed that this phase synchronization can solve the binding problem for figure-ground segregation (von der Malsburg and Schneider 1986) and underlie visual attention and awareness (Crick and Koch 1990). Second, synchronous bursts converging on a postsynaptic target cell will produce large depolarizations that are optimal for activating NMDA receptors leading to 
long-term potentiation (Brown et al. 1990). This suggests the possibility that the induction of plasticity requires temporal synchronization of synaptic input.

A number of theoretical explanations based on coupled (relaxation) oscillator models have been proposed for burst synchronization (Sompolinsky et al. 1989; Kammen et al. 1990). The crucial issue of phase synchronization has also recently been addressed by Bush and Douglas (1991), who simulated the dynamics of a network consisting of bursty, layer $\mathrm{V}$ pyramidal cells coupled to a common pool of basket cells inhibiting all pyramidal cells. ${ }^{1}$ The cells were modeled using Hodgkin and Huxley-like dynamics. Bush and Douglas found that excitatory interactions between the pyramidal cells increases the total neural activity as expected and that global inhibition leads to synchronized bursts with random intermissions. These population bursts appear to occur in a random manner in their model. The basic mechanism for the observed burst synchronization is hidden in the numerous anatomical and biophysical details of their model.

These, and the related observation that to date no strong oscillations have been recorded in the neuronal activity in visual cortex of awake monkeys, prompted us to investigate how phase synchronization can occur in the absence of frequency locking. We proceed by replacing the cortical architecture of Bush and Douglas (1991) by a simple, exactly solvable model of all-to-all, excitatory coupled binary McCulloch-Pitts neurons (1943) that are globally connected to one inhibitor that we simulate by an activity-dependent common threshold. We find that for random uncorrelated inputs the output of the network consists of synchronized bursts with seemingly random intermissions. This shows that burst synchronization is a generic feature of such a neuronal architecture, which amplifies incoming coincident signals to synchronous bursts.

Whenever several input signals coincide, they excite the network to a global burst of activity that is subsequently shut down by the inhibition. The minimal number of coincidences that is needed to trigger collective bursting increases with increasing $\theta / \omega$, where $\theta$ is the threshold of the neurons and $\omega$ measures the strength of the excitatory coupling. For $\theta / \omega \rightarrow 0$ the interburst interval decreases until one sees only a regular sequence of global bursts each followed immediately by zero activity. Therefore, the output of the network varies from essentially randomly separated synchronous bursts (for $\theta / \omega \lesssim 1$ ) to regular series of on-off activity (for $\theta / \omega \rightarrow 0$ ). To substantiate these statements, we analyze the fraction $m$ of synchronously firing neurons as a function of the random input activity. We show that the autocorrelation of the neuronal activity $m$ displays, in addition to a peak at zero time indicating random bursts, a tail that decays exponentially in an oscillatory fashion. The origin of this

\footnotetext{
'This model bears similarities to Wilson and Bower's (1991) model describing the origin of phase locking in olfactory cortex.
} 
damped oscillation can be traced back to the global inhibitory feedback. For $\theta / \omega \lesssim 1$, these oscillations are very strongly damped, nevertheless the network displays a periodic synchronous bursting whose interburst intervals are independent of the oscillatory period. This means that these oscillations play no constructive role for burst synchronization.

\section{A Coincidence Network}

We consider $n$ excitatory coupled binary McCulloch-Pitts (1943) neurons whose output $x_{i}^{t+1} \in[0,1]$ at time $t+1$ is given by

$$
x_{i}^{t+1}=\sigma\left[\frac{\omega}{n} \sum_{i} x_{i}^{t}+\xi_{i}^{t}-\theta\right]
$$

Here $\omega / n>1$ is the normalized excitatory all-to-all synaptic coupling, $\xi_{i}^{t}$ represents the external binary input, and $\sigma[z]$ is the Heaviside step function, such that $\sigma[z]=1$ for $z>0$ and 0 elsewhere. Each neuron has the same dynamic threshold $\theta>0$. Next we introduce the fraction $m^{t}$ of neurons that fire simultaneously at time $t$ :

$$
m^{t}=\frac{1}{n} \sum_{i} x_{i}^{t}
$$

In general, $0 \leq m^{t} \leq 1$; only if every neuron is active at time $t$ do we have $m^{t}=1$. By summing equation 2.1 we then obtain the following equation of motion for our simple network:

$$
m^{t+1}=\frac{1}{n} \sum_{i} \sigma\left[\omega m^{t}+\xi_{i}^{t}-\theta\right]
$$

The behavior of this finite-state automata (it can take on all the $n+1$ states characterized by $m^{t}=i / n$, with $\left.0 \leq i \leq n\right)$, is then fully described by the phase-state diagram of Figure 1. If $\theta>1$ and $\theta / \omega>1$, then the output of the network $m^{t}$ will vary with the input until at some time $t^{\prime}, m^{t^{\prime}}=0$. Since the threshold $\theta$ is now always larger than the input, the network will remain in this state, that is, $m^{t^{\prime \prime}}=0$, for all $t^{\prime \prime}>t^{\prime}$. If, on the other hand, the threshold $\theta<1$ and smaller than the weight, that is, $\theta / \omega<1$, the network will drift until it comes to the state $m^{t^{\prime}}=1$. Since from then on $\omega m^{t}$ is at all times larger than the threshold, the network remains latched at $m^{t}=1$. If $\theta>1$, but $\theta / \omega<1$, the network can latch in either the $m^{t}=0$ or the $m^{t}=1$ state and will remain there indefinitely. Lastly, if $\theta<1$, but $\theta / \omega>1$, the threshold is by itself not large enough to keep the network latched into the $m^{t}=1$ state. If we define the normalized input activity or noise

$$
s^{t}=\frac{1}{n} \sum_{i} \xi_{i}^{t}
$$




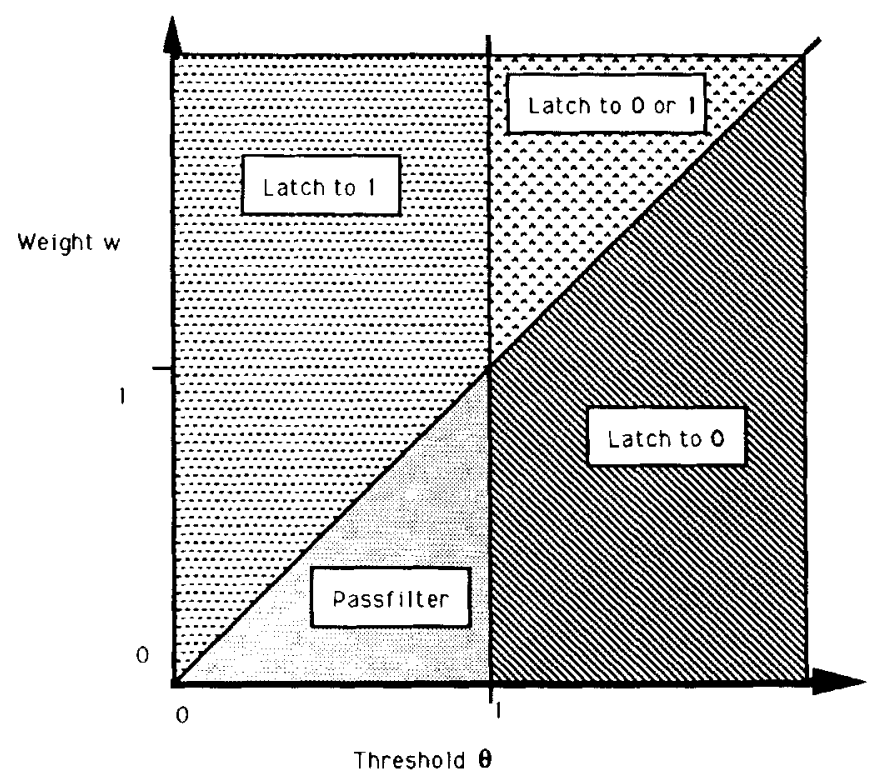

Figure 1: Phase diagram for the network described by equation 2.3. Different regions correspond to different stationary output states $m^{\prime}$ in the long time limit. For details see text.

with $0 \leq s^{t} \leq 1$, we see that in this part of phase space $m^{t+1}=s^{t}$; in other words, the total output activity faithfully reflects the input activity at the previous time step.

Let us now increase the behavioral repertoire of our network by introducing an adaptive time-dependent threshold, $\theta^{t}$, motivated by the use of global inhibition in Bush and Douglas (1991). We assume that $\theta^{t}$ remains at its value $\theta<1$ as long as the total activity remains less than 1 . If, however, $m^{t}=1$, we increase $\theta^{t}$ to a value larger than $\omega+1$. This has the effect of resetting the activity of the entire network to 0 in the next time step, that is, $m^{i+1}=(1 / n) \sum_{i} \sigma\left[\omega+\xi_{i}-(\omega+1+\epsilon)\right]=0$. The threshold will then automatically reset itself to its old value. In other words, we will now consider the case of

$$
m^{t+1}=\frac{1}{n} \sum_{i} \sigma\left[\omega m^{t}+\xi_{i}^{t}-\theta\left(m^{t}\right)\right]
$$

with

$$
\theta\left(m^{t}\right)= \begin{cases}\theta<1 & \text { for } m^{t}<1 \\ >\omega+1 & \text { for } m^{t}=1\end{cases}
$$


Therefore, we are operating in the topmost left part of Figure 1 but preventing the network from latching to $m^{t}=1$ by resetting it. Such a dynamic threshold bears some similarities to the models of Horn and Usher $(1989,1990)$, Treves and Amit (1989), and others, but is much simpler. Note that $\theta\left(m^{t}\right)$ exactly mimics the effect of a common inhibitory neuron that is excited only if all neurons fire simultaneously.

Our network now acts as a coincidence detector, such that all neurons will "fire" at time $t+2$, that is, $x_{i}^{t+2}=1$, for all $i$, and $m_{1}^{t+2}=1$ if at least $k$ neurons receive at time $t$ a " 1 " as input. $k$ is the smallest integer with $k>\theta \cdot n / \omega$. If the network receives at least $k$ such inputs, the network will react to this two time steps later by discharging all neurons, with $m^{t+2}=1$. The threshold $\theta\left(m^{t}\right)$ is then transiently increased and the network is reset and the game begins anew. In other words, the network detects coincidences and signals this by a synchronized burst of neuronal activity followed by a brief respite of activity. Figure 2 shows the typical behavior of our network.

The time dependence of $m^{t}$ given by equation 2.5 can be written as

$$
m^{t+1}= \begin{cases}s^{t} & \text { for } 0 \leq m^{t}<\theta / \omega \\ 1 & \text { for } \theta / \omega \leq m^{t}<1 \\ 0 & \text { for } m^{t}=1\end{cases}
$$

By introducing functions $A(m), B(m), C(m)$, which take on the value 1 in the intervals specified for $m=m^{t}$ in equation 2.6, respectively, and zero elsewhere (see also Fig. 3), we find that $m^{t+1}$ can be written as:

$$
m^{t+1}=s^{t} A\left(m^{t}\right)+1 \cdot B\left(m^{t}\right)+0 \cdot C\left(m^{t}\right)
$$

This equation can be iterated, yielding an explicit expression for $m^{t}$ as a function of the external inputs $s^{t+1}, \ldots, s^{0}$ and the initial value $m^{0}$ :

$$
m^{t}=\left(s^{t-1}, 1,0\right) M\left(s^{t-2}\right) \ldots M\left(s^{0}\right)\left(\begin{array}{c}
A\left(m^{0}\right) \\
B\left(m^{0}\right) \\
C\left(m^{0}\right)
\end{array}\right)
$$

with the matrix

$$
M(s)=\left(\begin{array}{ccc}
A(s) & 0 & 1 \\
B(s) & 0 & 0 \\
C(s) & 1 & 0
\end{array}\right)
$$

Equation 2.8 is the principal result of this section. It shows that the dynamics of our network model can be solved explicitly, by iteratively applying $M$, the transformation matrix, $t-1$ number of times to the initial network configuration.

\section{Distribution of Bursts and Time Correlations}

We have seen in the previous section in equation 2.8 that the synchronous activity at time $t$ depends on the specific realization of the input signals 

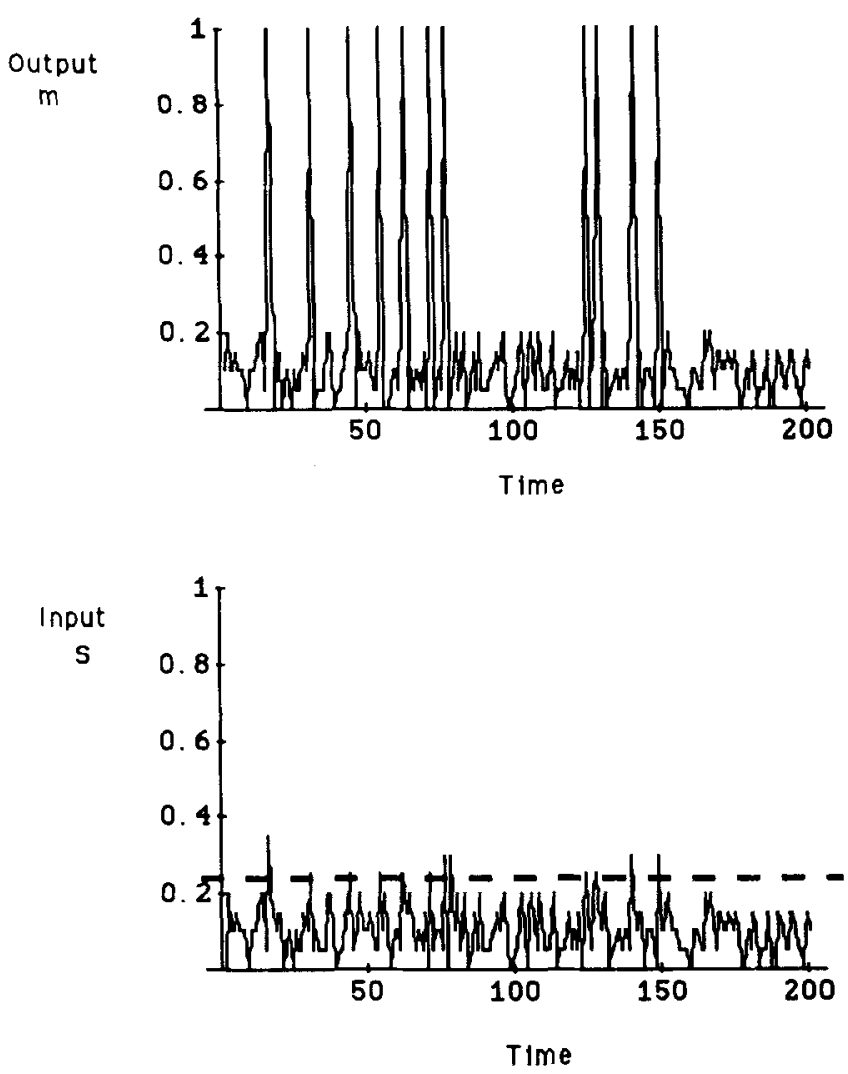

Figure 2: Time dependence of the fraction $m^{t}=(1 / n) \sum_{i} x_{i}^{t}$ of output neurons that fire simultaneously compared to the corresponding fraction of input signals $s^{t}=(1 / n) \sum_{i} \xi_{i}^{t}$, for $n=20$ and $\theta / \omega=0.225$. The input variables $\xi_{i}^{t}$ are independently distributed according to $P\left(\xi_{i}^{t}\right)=p \delta\left(\xi_{i}^{t}-1\right)+(1-p) \delta\left(\xi_{i}^{t}\right)$ with $p=0.1$. One sees that only coincidences with $s^{l}>0.225$ (dotted line; this corresponds to at least five input signals with $\xi_{i}^{t}=1$ ) lead to the entire population firing in synchrony two time steps later, that is, $m^{t+2}=1$. Note the "random" appearance of the interburst intervals. All simulations were carried out using MATHEMATICA.

at different times. To get rid of this ambiguity we have to resort to averaged quantities where averages are understood over the distribution $\hat{P}\left\{s^{t}\right\}$ of inputs $s^{t}=1 / n \sum_{i=1}^{n} \xi_{i}^{t}$. A very useful averaged quantity is the probability $P^{t}(m)$, describing the fraction $m$ of simultaneously firing 


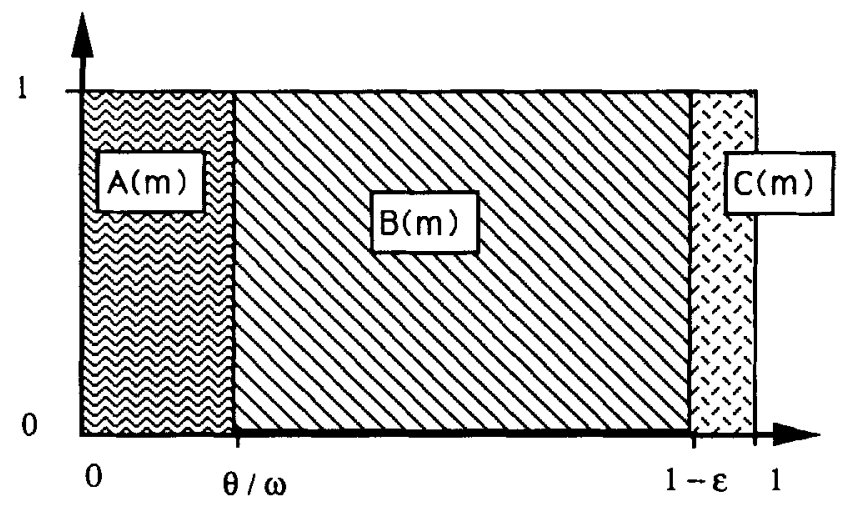

m

Figure 3: Regions on the $m$-axis when the functions $A(m), B(m)$, and $C(m)$ have value 1 . Outside these regions all these functions are zero.

neurons at time $t . P^{t}(m)$ is related to the probability distribution $P\left\{s^{t}\right\}$ via

$$
P^{t}(m)=\left\langle\delta\left[m-m^{t}\left\{s^{t-1} \ldots s^{0}\right\}\right]\right\rangle
$$

where $\langle\ldots\rangle$ denotes the average with respect to $\hat{P}\left\{s^{t}\right\}$ and $m^{t}\left\{s^{t-1}, \ldots, s^{0}\right\}$ is given by equation 2.8. If the input signals $\xi_{i}^{t}$ are uncorrelated in time, $m^{t+1}$ depends according to equation 2.7 only on $\mathrm{m}^{t}$, and the time evolution of $P^{t}(m)$ is described by the Chapman-Kolmogorov equation:

$$
P^{t+1}(m)=\int_{0}^{1} d m^{\prime} K\left(m \mid m^{\prime}\right) P^{t}\left(m^{\prime}\right)
$$

with the integral kernel

$$
\begin{aligned}
K\left(m \mid m^{\prime}\right) & =\int_{0}^{1} d s \hat{P}(s) \delta\left[m-s A\left(m^{\prime}\right)-B\left(m^{\prime}\right)-0 \cdot C\left(m^{\prime}\right)\right] \\
& =\hat{P}(m) A\left(m^{\prime}\right)+\delta(m-1) B\left(m^{\prime}\right)+\delta(m) C\left(m^{\prime}\right)
\end{aligned}
$$

Iteration of equations 3.2 and 3.3 yields:

$$
P^{t}(m)=[\hat{P}(m), \delta(m-1), \delta(m)] \bar{M}^{t-1} \mathbf{v}
$$

where

$$
\bar{M}=\langle M(s)\rangle=\left(\begin{array}{ccc}
1-\eta & 0 & 1 \\
\eta & 0 & 0 \\
0 & 1 & 0
\end{array}\right)
$$


and

$$
\eta=\int_{0}^{1} \hat{P}(s) B(s) d s=\int_{\theta / \omega}^{1} \hat{P}(s) d s
$$

Notice that $0 \leq \eta \leq 1$ holds. Here we used the facts that the distribution $\hat{P}(s)$ is normalized to unity, $\int_{0}^{1} \hat{P}(s)[A(s)+B(s)+C(s)] d s=1$, and $C(m)$ is only 1 at the point $m=1$, that is, $\int_{0}^{1} d s \hat{P}(s) C(s)=0$. The starting vector $\mathbf{v}$ is related to the initial distribution $P^{0}(u)$ via $\mathbf{v}=\left[\int_{0}^{1} d m A(m) P^{0}(m)\right.$, $\left.\int_{0}^{1} d m B(m) P^{0}(m), \int_{0}^{1} d m C(m) P^{0}(m)\right]$. Equations 3.4 and 3.5 can be solved in terms of the eigenvalues and eigenvectors of $\bar{M}$ and we find

$$
P^{t}(m)=P^{\infty}(m)+\left[P^{0}(m)-P^{\infty}(m)\right] \cdot f(t)
$$

where

$$
P^{\infty}(m)=\frac{1}{1+2 \eta}[\hat{P}(m)+\eta \delta(m-1)+\eta \delta(m)]
$$

is the limiting distribution which evolves from the initial distribution $P^{0}(m)$ for large times, because the factor $f(t)=\eta^{t / 2} \cos (\Omega t)$, where

$$
\Omega=\pi-\arctan \left[\sqrt{4 \eta-\eta^{2}} / \eta\right]
$$

decays exponentially with time.

Equations 3.6 and 3.7 show that the limiting equilibrium distribution $P^{\infty}(m)$ evolves from the initial distribution $P^{0}(m)$ in an oscillatory fashion, with the building up of two delta functions at $m=1$ and $m=0$ at the expense of $P^{0}(m)$. This signals the emergence of synchronous bursts, that is, $m^{t}=1$, which are always followed at the next time step by zero activity, that is, $m^{t+1}=0$ (see also Fig. 2). The mean fraction $\left\langle m^{t}\right\rangle=$ $\int_{0}^{1} d m P^{t}(m) m$ of synchronized neurons evolves as

$$
\left\langle m^{t}\right\rangle=\left\langle m^{\infty}\right\rangle-\left[\left\langle m^{0}\right\rangle-\left\langle m^{\infty}\right\rangle\right] f(t)
$$

We obtain from equation 3.7 that the equilibrium value

$$
\left\langle m^{\infty}\right\rangle=\int_{0}^{1} d m P^{\infty}(m) m=\frac{\langle s\rangle+\eta}{1+2 \eta}
$$

which is larger than the initial value $\langle s\rangle=\int_{0}^{1} d s P(s) s$, for $\langle s\rangle\langle 1 / 2$, indicating an increase in synchronized bursting activity.

We saw that the equilibrium state of the system is approached in an oscillatory fashion. It is therefore interesting to ask what type of time correlations will develop in the output of our network if it is stimulated with uncorrelated noise, $\xi_{i}^{t}$. The time correlation function, also known as the autocovariance (since we subtract the mean activity)

$$
C(\tau)=\lim _{t \rightarrow \infty}\left[\left\langle m^{t+\tau} m^{t}\right\rangle-\left\langle m^{t}\right\rangle^{2}\right]
$$


can be computed directly since $m^{t}$ and $P^{\infty}(m)$ are known explicitly from equations 3.7 and 3.8 . We find

$$
C(\tau)=\delta_{\tau, 0} C_{0}+\left(1-\delta_{\tau, 0}\right) C_{1} \eta^{|\tau| / 2} \cos (\Omega \tau+\varphi)
$$

with $\delta_{\tau, 0}$ the Kroneker symbol, with $\delta_{\tau, 0}=1$ for $\tau=0$ and 0 else. ${ }^{2}$ Figure 4 shows that $C(\tau)$ from equation 3.10 consists of two parts. A delta peak at $\tau=0$ that reflects random uncorrelated bursting and an oscillatory decaying part that indicates correlations in the output. The period of the oscillations

$$
T=\frac{2 \pi}{\Omega}=\frac{2 \pi}{\pi-\arctan \left[\sqrt{4 \eta-\eta^{2}} / \eta\right]}
$$

varies monotonically between $3 \leq T \leq 4$ as $\theta / \omega$ moves from zero to one. Since $\eta$ is given by $\int_{\theta / \omega}^{1} \hat{P}(s) d s$, we see that the strengths of these oscillations increase as the excitatory coupling $\omega$ increases. The emergence of periodic correlations can be understood in the limit $\theta / \omega \rightarrow 0$, where the period $T$ becomes three (and $\eta=\int_{0}^{T} \hat{P}(s) d s=1$ ), because according to equation 2.6, $m^{t}=0$ is followed by $m^{t+1}=s^{t}$, which leads for $\theta / \omega \rightarrow 0$ always to $m^{t+2}=1$ followed by $m^{t+3}=0$. In other words, the temporal dynamics of $m^{t}$ has the form $0 s^{1} 10 s^{4} 10 s^{7} 10 s^{10} 10 \ldots$. In the opposite case of $\theta / \omega \rightarrow 1, \eta$ converges to 0 and the autocovariance function $C(\tau)$ essentially contains the peak only at $\tau=0$. Thus, the output of the network ranges from completely uncorrelated noise for $\theta / \omega \approx 1$ to correlated periodic bursts for $\theta / \omega \rightarrow 0$. Figure 4 shows the correlation function for two intermediate situations.

The amplitude of the Fourier transform of the autocovariance function $C(\tau)$, that is the power spectrum of the system, has the form

$$
P\left(\omega_{1}\right)=C_{0}+\frac{2 C_{1} \alpha}{\alpha^{2}+\left(\omega_{t}-\Omega\right)^{2}}
$$

with $\alpha=-\log \eta / 2$. In other words, a broad Lorentzian centered at the oscillation frequency, superimposed onto a constant background corresponding to uncorrelated neural activity.

It is important to discuss in this context the effect of the size $n$ of the network. If the input variables $\xi_{i}^{l}$ are distributed independently in time and space with probabilities $\tilde{P}_{i}\left(\xi_{i}^{t}\right)$, then the distribution $\hat{P}(s)$ has a width $^{3}$ which decreases as $1 / \sqrt{n}$ as $n \rightarrow \infty$. Therefore, in a large system $\eta=\int_{\theta / \omega}^{1} \hat{P}(s) d s$ is either 0 if $\left.\theta / \omega\right\rangle\langle s\rangle$ or 1 if $\theta / \omega<\langle s\rangle$, where $\langle s\rangle$ is the

${ }^{2}$ The constants $C_{0}, C_{l}$ and $\varphi$ can be determined from $C(\tau)=\delta_{\tau, 0}\left\langle m^{2}\right\rangle_{\infty}+(1-$ $\left.\delta_{\tau, 0}\right)[\langle s\rangle, 1,0] M^{\tau-1} \mathbf{b}-\langle m\rangle_{\infty}^{2}$, where $\mathbf{b}=\left[\langle A(m) m\rangle_{\infty},\langle B(m) m\rangle_{\infty},\langle C(m) m\rangle_{\infty}\right]$ and $\langle\ldots\rangle_{\infty}$ denotes the average over $P^{\infty}(m)$. For $\eta=0$ this yields $C_{0}=\left\langle s^{2}\right\rangle-\langle s\rangle^{2} ; C_{1}=C_{2}=0$ and for $\eta=1, \hat{P}(s)=\delta(s-p), p>\theta / \omega$ with $C(0)=C_{0}=\left(p^{2}+1\right) / 3-(p+1)^{2} / 9, C(1)=$ $p / 3-(p+1)^{2} / 9, C(2)=C(1), C(3)=C(0)$, that is, an oscillation with period 3 .

${ }^{3}$ The mean squared fluctuation is $\left\langle s^{2}\right\rangle-\langle s\rangle^{2}=\int d \xi_{1}^{t} \ldots \int d \xi_{n}^{t} \tilde{P}_{1}\left(\xi_{1}^{t}\right) \ldots \tilde{P}_{n}\left(\xi_{n}^{t}\right)\left(\frac{1}{n} \sum_{i=1}^{n} \xi_{i}^{t}\right)^{2}$ $-\left[\int d \xi_{1}^{t} \ldots \int d \xi_{n}^{t} \tilde{P}_{1}\left(\xi_{1}^{t}\right) \ldots \tilde{P}_{n}\left(\xi_{n}^{t}\right)\left(1 / n \sum_{i=1}^{n} \xi_{i}^{t}\right)\right]^{2} \propto \frac{1}{n}$ for $n \rightarrow \infty$. 

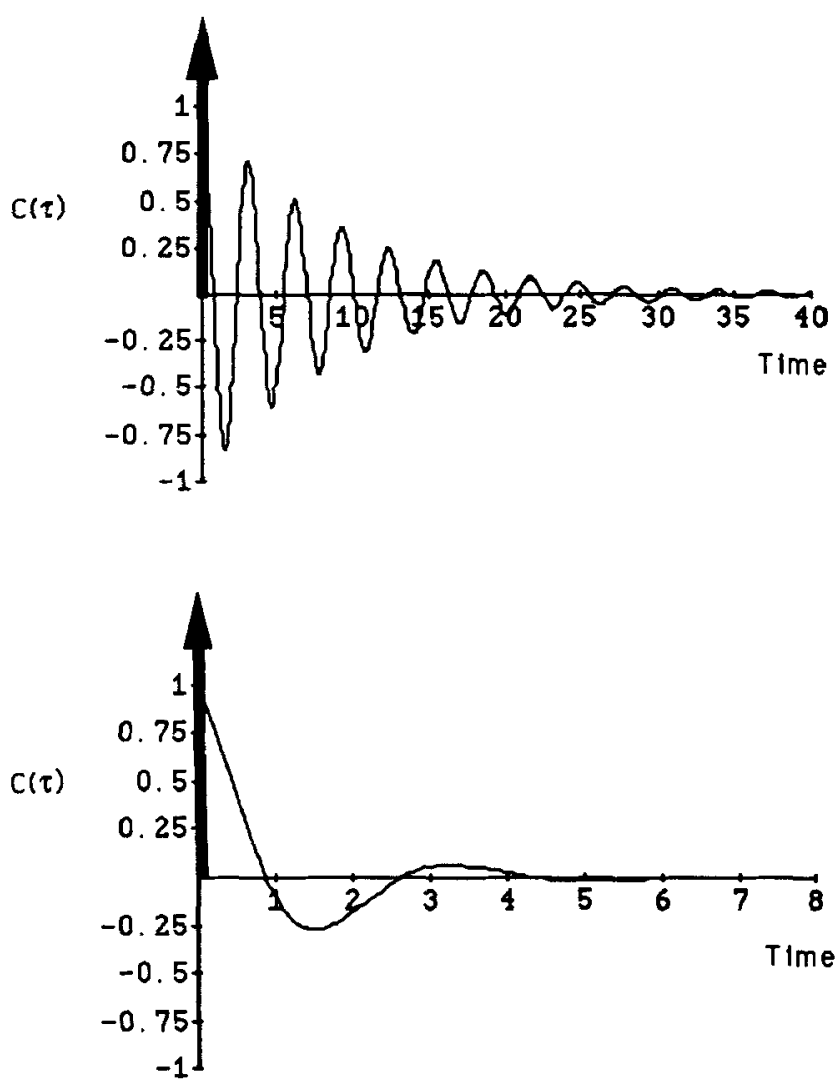

Figure 4: Time dependence of the autocorrelation function $C(\tau)=$ $\lim _{t \rightarrow \infty}\left\{\left\langle m^{t+\tau} m^{t}\right\rangle-\left\langle m^{t}\right\rangle^{2}\right\}$ for two different values of $\eta=\int_{\theta / \omega}^{1} d s \hat{P}(s)$. The top figure corresponds to $\eta=0.8$ and a period $T=3.09$; the bottom correlation function is for $\eta=0.2$ with an associated $T=3.50$. Note the different time scales.

mean value of $s$, which coincides for $n \rightarrow \infty$ with the maximum of $\hat{P}(s)$. If $\eta=0$ the correlation function is a constant according to equation 3.11, while the system will exhibit undamped oscillations with period 3 for $\eta=1$. An earlier example where the mean activity of a large population of neurons converges either to a fixed point or to a limit cycle has been discussed by Sompolinsky (1988). Therefore, the irregularity of the burst intervals, as shown, for instance, in Figure 2, is for independent $\xi_{i}^{\dagger}$ a finite 
size effect. Such synchronized dephasing due to finite size has already been reported by Sompolinsky et al. (1989).

However, for biologically realistic correlated inputs $\xi_{i}^{t}$, the width of $\hat{P}(s)$ can remain finite for $n \gg 1$. For example, if the inputs $\xi_{1}^{t}, \ldots, \xi_{n}^{t}$ can be grouped into $q$ correlated sets $\xi_{1}^{\dagger} \ldots \xi_{1}^{\dagger}, \xi_{2}^{t} \ldots \xi_{2}^{t}, \ldots, \xi_{q}^{\dagger} \ldots \xi_{q}^{\dagger}$, with finite $q$, then the width of $\hat{P}(s)$ scales like $1 / \sqrt{q}$. Our model, which now effectively corresponds to a situation with a finite number $q$ of inputs, leads in this case to irregular bursts that mirror and amplify the correlations present in the input signals, with an oscillatory component superimposed due to the dynamic threshold.

\section{Conclusions and Discussion}

We here suggest a mechanism for burst synchronization that is based on the fact that excitatory coupled neurons fire in synchrony whenever a sufficient number of input signals coincide. In our model, common inhibition shuts down the activity after each burst, making the whole process repeatable. But the inhibition does not entrain signals in contrast to previous suggestions (e.g., Lytton and Sejnowski 1991). It is rather satisfactory to us that our simple model shows similarities to the dynamic behavior of the much more detailed biophysical simulations of Bush and Douglas (1991). They use in their model neurons that differ in their firing rates due to differences in cellular parameters. We use neurons with random input that generate random firing in the absence of strong coupling. In both models, all-to-all excitatory coupling leads, together with common inhibition, to burst synchronization without frequency locking. In our analysis we updated all neurons in parallel. The same model has been investigated numerically for serial (asynchronous) updating, leading to qualitatively similar results. Furthermore, very similar results should be obtained with the use of continuous neurons instead of our binary ones (Hopfield 1984).

The output of our network develops oscillatory correlations whose range and amplitude increase as the excitatory coupling is strengthened. However, these oscillations do not depend on the presence of any neuronal oscillators, as in our earlier models (e.g., Kammen et al. 1990; Schuster and Wagner 1990; Niebur et al. 1991). The period of the oscillations reflects essentially the delay between the inhibitory response and the excitatory stimulus and varies only little with the amplitude of the excitatory coupling and the threshold. The crucial role of inhibitory interneurons in controlling the $40 \mathrm{~Hz}$ neuronal oscillations has been emphasized by Wilson and Bower (1991) in their simulations of olfactory and visual cortex. Our model shows complete synchronization, in the sense that all neurons fire at the same time. This suggests that the occurrence of tightly synchronized firing activity across neurons (Freeman 1978; Eckhorn et al. 1988; Gray et al. 1989; Engel et al. 1990; Wilson and Bower 1991) is more 
important for feature linking and binding than the locking of oscillatory frequencies. Since the specific statistics of the input noise is, via coincidence detection, mirrored in the burst statistics, we speculate that our network - acting as an amplifier for the input noise - can play an important role in any mechanism for feature linking that exploits common noise correlations of different input signals.

\section{Acknowledgments}

We thank R. Douglas for stimulating discussions and for inspiring us to think about this problem and $\mathrm{H}$. Sompolinsky for pointing out the importance of finite size effects. Our collaboration was supported by the Stiftung Volkswagenwerk. The research of C. K. is supported by the National Science Foundation, the James McDonnell Foundation, and the Air Force Office of Scientific Research.

\section{References}

Brown, T. H., Kairiss, E. W., and Keenan, C. L. 1990. Hebbian synapses: Biophysical mechanisms and algorithms. Annu. Rev. Neurosci. 13, 475-511.

Bush, P. C., and Douglas, R. J. 1991. Synchronization of bursting action potential discharge in a model network of neocortical neurons. Neural Comp. 3, 19-30.

Crick, F., and Koch, C. 1990. Towards a neurobiological theory of consciousness. Semin. Neurosci. 2, 263-275.

Eckhorn, R., Bauer, R., Jordan, W., Brosch, M., Kruse, W., Munk, M., and Reitboeck, H. J. 1988. Coherent oscillations: A mechanism of feature linking in the visual cortex? Biol. Cybern. 60, 121-130.

Engel, A. K., König, P., Gray, C. M., and Singer, W. 1990. Stimulus-dependent neuronal oscillations in cat visual cortex: Inter-columnar interaction as determined by cross-correlation analysis. Eur. J. Neurosci. 2, 586-606.

Freeman, W. J. 1978. Spatial properties of an EEG event in the olfactory bulb and cortex. Electroencephalogr. Clin. Neurophysiol. 44, 586-605.

Gray, C. M., Engel, A. K., König, P., and Singer, W. 1990. Stimulus-dependent neuronal oscillations in cat visual cortex: Receptive field properties and feature dependence. Eur. J. Neurosci. 2, 607-619.

Gray, C. M., König, P., Engel, A. K., and Singer, W. 1989. Oscillatory response in cat visual cortex exhibits inter-columnar synchronization which reflects global stimulus attributes. Nature (London) 338, 334-337.

Hopfield, J. J. 1984. Neurons with graded response have collective computational properties like those of two-state neurons. Proc. Natl. Acad. Sci. U.S.A. 81, 3088-3092.

Horn, D., and Usher, M. 1989. Neural networks with dynamical thresholds. Phys. Rev. A 40, 1036.

Horn, D., and Usher, M. 1990. Excitatory-inhibitory networks with dynamical thresholds. Int. J. Neural Syst. 1, 249-257. 
Kammen, D., Holmes, P., and Koch, C. 1990. Collective oscillations in neuronal networks. In Advances in Neural Information Processing Systems, Vol. 2, D. Touretzky, ed., pp. 76-83. Morgan Kaufmann, San Mateo, CA.

Lytton, W. W., and Sejnowski, T. J. 1991. Simulations of cortical pyramidal neurons synchronized by inhibitory interneurons. J. Neurophysiol. 66, 1-22.

McCulloch, W. S., and Pitts, W. A. 1943. A logical calculus of the ideas immanent in neural nets. Bull. Math. Biophys. 5, 115-137.

Niebur, E., Kammen, D. M., Koch, C., Ruderman, D., and Schuster, H. G. 1991. Phase-coupling in two-dimensional networks of interacting oscillators. In Advances in Neural Information Processing Systems, D. S. Touretzky, and R. Lippman, eds., pp. 123-129. Morgan Kaufmann, San Mateo, CA.

Schuster, H. G., and Wagner, P. 1990. A model for neuronal oscillations in the visual cortex: I Mean-field theory and the derivation of the phase equations. Biot. Cybern. 64, 77-82.

Sompolinsky, H. 1988. Statistical mechanics of neural networks. Phys. Today 40, 2-12.

Sompolinsky, H., Golomb, D., and Kleinfeld, D. 1989. Global processing of visual stimuli in a neural network of coupled oscillators. Proc. Natl. Acad. Sci. U.S.A. 87, 7200-7204.

Sporns, O., Gally, J. A., Reeke, G. N., Jr., and Edelman, G. M. 1989. Reentrant signalling among simulated neuronal groups leads to coherency in their oscillatory activity. Proc. Natl. Acad. Sci. U.S.A. 86, 7265-7269.

Treves, A., and Amit, D. 1989. Low firing rates: An effective Hamiltonian for excitatory neurons. J. Phys. A. 22, 2205-2226.

von der Malsburg, C., and Schneider, W. 1986. A neural cocktail-party processor. Biol. Cybern. 54, 29-40.

Wilson, M. A., and Bower, J. M. 1992. Cortical oscillations and temporal interactions in a computer simulation of piriform cortex. J. Neurophysiol., in press.

Received 2 April 1991; accepted 26 August 1991. 